\title{
Plymouth students help empower local families
}

Year 2 undergraduate students at Peninsula Dental School, University of Plymouth (UoP), undertake an 'Inter-professional engagement' as part of their dental training. The module, which is coordinated by the community health and wellbeing charity Well Connected, enables dental and dental therapy and hygiene students to work with a community organisation to design and deliver a project that promotes healthy lifestyles and behaviours. The module also encourages students to consider the personal, community and wider determinants of health of their target population.

For the academic year 2018-19, a group of dental hygiene students (pictured with their academic supervisor, the community engagement programme officer and the oral health educator) were assigned to work with the Family Intensive Intervention Project (FIIP). FIIP is run by the Local Authority (Plymouth City Council) and aims to provide support to families who are dealing with social, economic, health and behaviour hardships.

The aim of the project was to improve oral health knowledge amongst FIIP families and key workers to empower families to make informed choices. The objectives for this project were to:

- Provide information on appropriate toothbrushes and fluoride concentrations for specific ages using a 'fluoride mini guide'

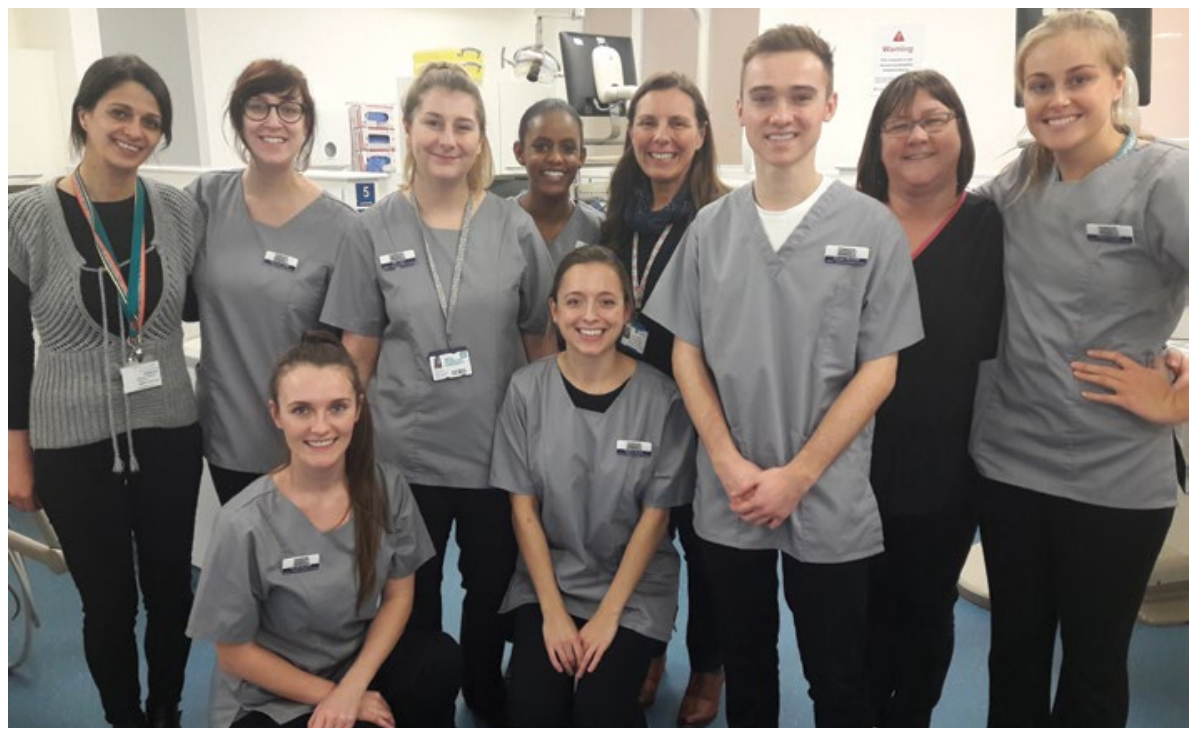

- Raise awareness of sugar content of popular foods using the 'Change 4 Life app' and highlight the daily sugar allowance for specific ages using a 'Sugar Passport'

- Educate families about the link between bacteria, oral disease and general health by highlighting the importance of effective hand washing

- Produce a beneficial aide-mémoire for keyworkers, to support the delivery of key health messages to future FIIP families.

The above objectives were met through the delivery of an interactive and engaging workshop that took place at the Dental
Education Facility (UoP). The oral hygiene station was implemented through talking animated animals, created using the google app Chudo. Each of the animals had been given a name (for example Bella the Bunny and Terry the Tiger), making the oral hygiene station fun, memorable and engaging for all involved. The messages delivered were based on Delivering better oral health guidelines by Public Health England.

The presentation consisting of the animated animals and their messages is now being used by the charity Well Connected as a means of promoting oral health messages to young children.

\section{BOOK REVIEW}

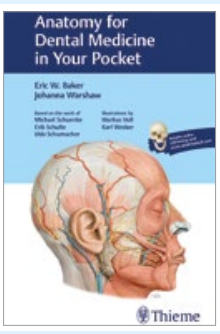

This new addition to the huge array of anatomical textbooks is noteworthy for its targeted synoptic selection of structures relevant to dental and allied professional students and health practitioners. Its wirebound format and compact dimensions make it ideal for 'in your pocket' portability. Furthermore, easy page selection and the high quality glossy thick paper on which it is printed make it an accomplished stain-resistant accompaniment for dissection laboratory usage.

The 153 full colour illustrations are arranged as cards with key illustrated structures labelled numerically and lead lines to named structures correlated on the reverse side of the card. This arrangement makes this book an ideal rapid reference resource.
The whole of the head and neck anatomy is covered in 13 chapters devoted to regions ranging from the skull to the neck, face, eyes, ears, nose, and lymphatics; ending with clinical notes on potential spaces for the spread of infection. Even for clinicians, this compact book serves to facilitate as an aide-mémoire to anatomical identities of nerves, vessels and regions of the head and neck. The online self-testing tool is an added bonus to reinforce recollection and memorising of dissecting room learning.

The remarkably reasonable price makes it accessible to even cash-strapped students. In all, a highly recommended learning tool.

G. H. Sperber 